\title{
Porous organic cage membranes for biofuel purification via pervaporation: A molecular simulation study
}

Jie Liu ${ }^{\text {a }}$ Lin $\mathrm{Li}^{\text {a }}$, Zitong Zhao ${ }^{\text {a }}$, and Faquan $\mathrm{Yu}^{\mathrm{a}^{*}}$

${ }^{a}$ Key Laboratory for Green Chemical Process of Ministry of Education, Hubei Key Laboratory for

Novel Reactor and Green Chemistry Technology, Hubei Engineering Research Center for Advanced

Fine Chemicals, School of Chemical Engineering and Pharmacy, Wuhan Institute of Technology,

Wuhan 430205, China

*Email: fyu@wit.edu.cn, fyuwucn@gmail.com

Total number of pages: 3

Total number of figures:3 
(a) $\mathrm{CC} 3$

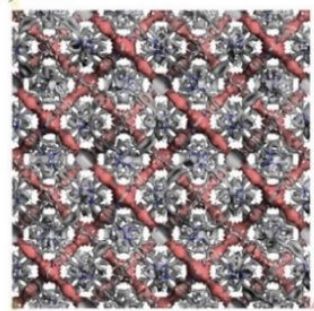

(d) $\mathrm{CC} 5$

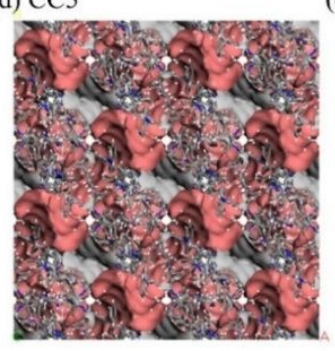

(b) $\mathrm{CC} 19$

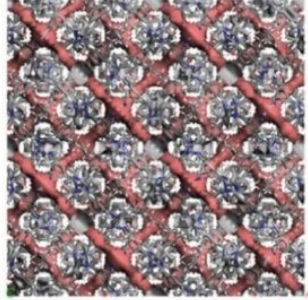

(e)

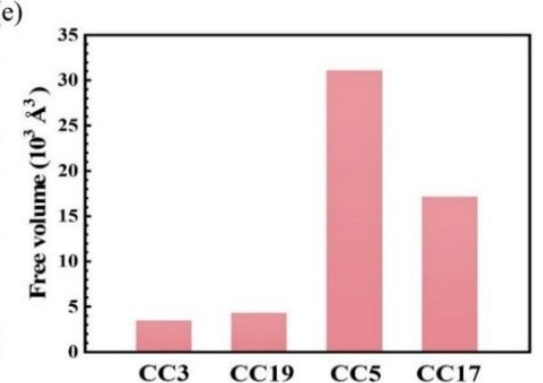

Figure S1. Pore structures of (a) CC3, (b) CC19, (c) CC17, (d) CC5. The membrane skeleton is presented in gray and the pore structure is rendered in pink. (e) Free volumes of the four POC membranes.

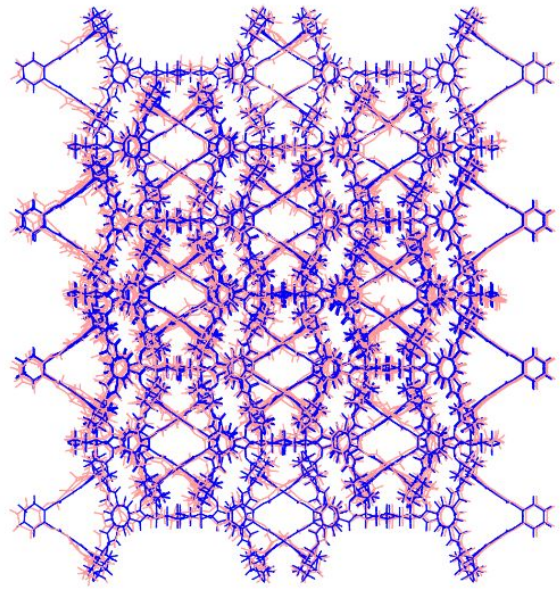

Figure S2. Superimposed structure of flexible model (pink) on rigid model (blue) after pervaporation. 


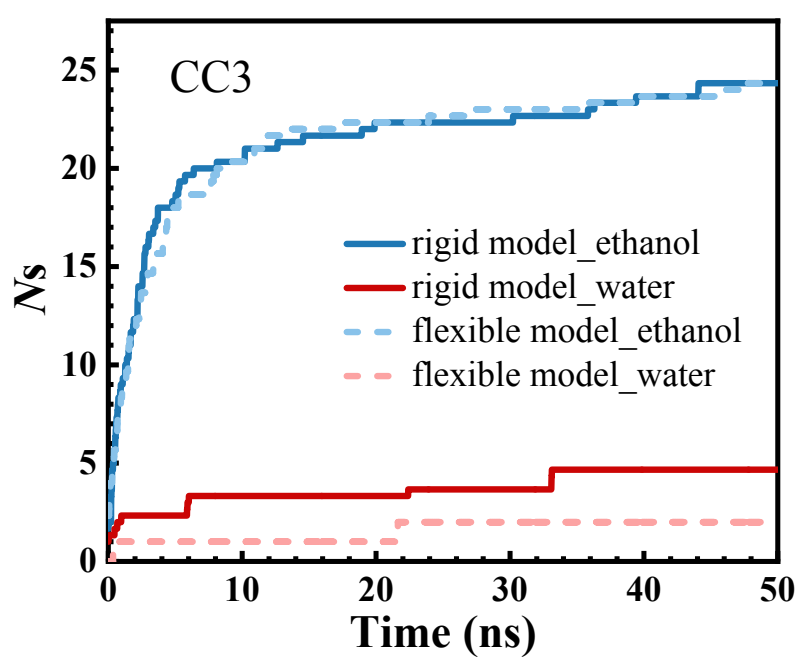

Figure S3. Ethanol and water flows through CC3 membranes for ethanol/water mixtures $\left(x_{e}=10 \mathrm{wt} \%\right)$ in two different models. 